\title{
Color Radiomap Interpolation for Efficient Fingerprint WiFi-based Indoor Location Estimation
}

\author{
Kohei Arai \\ Information Science Department \\ Saga University \\ Saga, Japan
}

\author{
Herman Tolle \\ Information System Department, \\ Brawijaya University \\ Malang, Indonesia
}

\begin{abstract}
Indoor location estimation system based on existing 802.11 signal strength is becoming increasingly prevalent in the area of mobility and ubiquity. The user-based location determination system utilizes the information of the Signal Strength (SS) received from the surrounding Access Points (APs) to determine the user position. In this paper, we focus on the development of a user position estimation using existing WiFi environment for its low cost and ease of deployment and study fingerprint-based deterministic techniques for their simplicity and effectiveness. We present the color radio map interpolation method with ease to development, reduce the calibration effort on creating radio map while still retain the accuracy of user position estimation. The average accuracy error 1.108 meter is achieved on 1.25 meter $x 2.5$ meter of cell grid size.
\end{abstract}

Keywords—network; indoor localization; radio map; user monitoring, location estimation

\section{INTRODUCTION}

In the era of ubiquitous computation, location-aware system becoming increasingly popular as well as practical. Knowing the locations of a device and the user inside a building is a necessary prerequisite for location-based services. Location estimation systems can be classified regarding various parameters: indoor or outdoor, hardware dependent or independent, deterministic or probabilistic based on the technique, WiFi, Bluetooth, infrared, ultrasound, and ultrawideband depending on the technology. One promising approach to measuring location is triangulation from 802.11 signal strength on wireless devices. Given radio signal strength measurements on the client from a few different access points, researchers have shown how to compute location down to a few meters. This type of location measurement is especially attractive because it only uses the buildings and users existing devices. We can use existing wireless network in a building that communicate with users mobile phone devices. This system works properly in indoors where GPS and mobile phone location is not working.

However, the accuracy of such systems usually depends on a meticulous calibration procedure that consists of physically moving a wireless client to many different known locations, and sometimes orientations, inside a building. It may be unrealistic to expect anyone to spend the resources on such work. When presented with this prospect as part of a new product, software product planners sometimes balk, complaining that system administrators are reluctant to keep the locations of printers updated, much less create and maintain a high-resolution table of 802.11 signal strengths.
One alternative to manual calibration is to analytically predict signal strengths based on a real physical simulation of the building and radio frequency propagation. There is a work on predicting signal strengths for wireless networking [1][2], but mostly aimed as a guide to the placement of access points and not location measurement. Bahl and Padmanabhan's RADAR [3] system was one of the first and most comprehensive studies of 802.11 location, and they considered the question of physical simulation versus manual calibration of signal strengths. They discovered that physically simulating signal strengths increased their median location error by about $46 \%$ (from 2.94 meters to 4.3 meters) over manual calibration. Moreover, a good physical simulation usually requires a more detailed model of the building than is normally available.

Many researchers try to find the best method to overcome the problem of calibration effort. They try to reduce the number of sample or physical area and interpolate to determine the value on that blank spot area [4][5]. This is more difficult with most other 802.11 location algorithms which instead must classify signal strengths into only previously seen locations. As expected, the accuracy goes down with reduced calibration data, so they should make tradeoff between accuracy and effort. In this paper, we focus on how to significantly reduce the offline calibration effort while still achieving high accuracy in location estimation with effective visualization capabilities. We proposed a color based radiomap interpolation where the interpolation is made by using color representative of signal strength information. The proposed system based on fingerprint radio map with initial cell grid size that improved with gradually color interpolation. Empirical experiment show that proposed system gain the high accuracy while improved the radio map grid size with color interpolation that reduce the effort on offline calibration.

\section{INDOOR LOCATION ESTIMATION BASED ON WIFI SigNAL STRENGTH}

\section{A. Positioning Techniques}

Bose and Heng [6] classified WiFi-based positioning methods into Cell Identity (Cell-ID), Time of Arrival (TOA), Time Difference of Arrival (TDOA), Angle of Arrival (AOA), and signal strength categories.

Cell Identity (Cell-ID) is a basic wireless positioning system solution. It matches the target's position with its connection to an Access Point (AP). It does not require complex operations such as time synchronization and multiple APs. Time of Arrival (TOA) measures a distance using the 
travel time of a radio signal from a transmitter to a receiver. Its application requires time synchronization of the transmitter and receiver, which is difficult to achieve for close ranges. To overcome the problem, Time Difference of Arrival (TDOA) was developed, which utilizes the time difference between the receiver and two or more receivers. That is to say, whereas TOA requires time synchronization of transmitters and receivers, TDOA needs just synchronization between receivers. Angle of Arrival (AOA) determines the position of a receiver by measuring the angle to it from a transmitter. An AP must use smart antennas and be capable of mounting them under static conditions.

Signal Strength based technique uses the signal attenuation property of the radio wave Received Signal Strength Indication (RSSI) to measure the distance from a receiver to transmitter using the distance-to-signal-strength relationship. One common approach of RSSI-based system is fingerprint approach that consists two phases: a training phase and a tracking phase. In the training phase, the received signal strength information is filtered, interpolated, and eventually stored in a database as sample points. In the tracking phase, the position is determined by comparison with the received signal strength sample points stored in the database [10]. The accuracy of this system is a function of the sample points' sampling space, an estimation method and the structure of the database. However, such a method requires the time consuming on survey procedure or calibration process.

\section{B. Reducing Calibration Time}

Reducing calibration time is one of the methods to decrease manual effort. In recent years, most research with high accuracy calibrate large amount of data on a fixed location. It normally requires tens or even hundreds of samples to stabilize signal strength distributions. With constant calibration frequency, the ratio between calibration samples and time is linear. Therefore, reducing calibration time to half of the origin means that only $50 \%$ of samples can be collected.

\section{Linear Interpolation Method}

Linear interpolation is a method of determining an unknown point between two known points. The unknown point $(x, y)$, then could calculated from the two known points $\left(x_{o}, y_{o}\right)$ and $\left(x_{1}, y_{1}\right)$. Linear interpolation commonly is expressed in the form

$$
y=y_{o}+\left(x-x_{0}\right) *\left(y_{1}-y_{0}\right) /\left(x_{0}-x_{0}\right)
$$

Also, this kind of interpolation on set of data points $\left(x_{0}, y_{0}\right)$, $\left(x_{1}, y_{1}\right), \ldots,\left(x_{n}, y_{n}\right)$ can be defined as the concatenation of linear interpolations between point pairs. The result is in a continuous line. In the present study, the interpolation method was used to predict the signal strength of an unknown point between two known points after Kalman filtering process. First, the coordinates $(x, y)$ of an unknown point between two points were obtained by interpolation. After that, the signal strength of the unknown point was calculated by interpolation.

\section{COLOR RADIOMAP INTERPOLATION METHOD FOR REDUCING CALIBRATION EFFORT}

\section{A. System Architecture}

In this research, a WiFi-based positioning system based on the fingerprint method was developed. As mentioned, the system is configured for two phases: training and tracking. The objective of the training phase is to build a signal information database, named Color Radiomap because we use color as representative of the radio signal. Received signal data were arranged in computer memory according to the IDs of APs. The signal data of the selected APs are then used in the signal processing step, involving filtering and interpolation, in order to reduce the noise effect. The signal data thus modified are stored in the database and converted into its color representative in cell based map or grid based map. In the tracking phase, the target's location is calculated by comparing the signal patterns stored in the signal database in the training phase. The calculated position of target then appears on the drawn system map in grid based position. Data processing is summarized in Fig. 1.

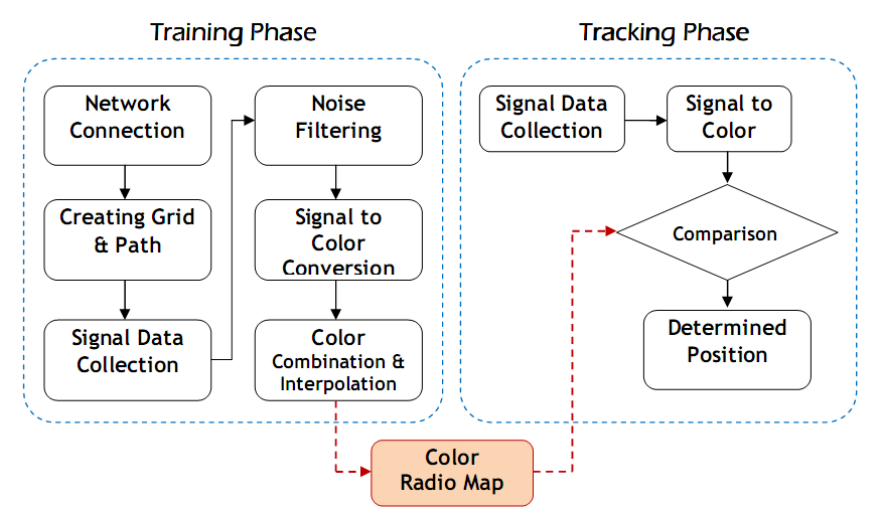

Fig. 1. Data Flow Of Indoor Location Estimation Fingerprint Method.

The data processing of each phase, in detail, is as follows.

\section{(1) Training phase}

The first step is to draw a two-dimensional map of the building for data collection and tracking. Based on the map, we create the grid in a rectangle shape and data gathering route is determined. Each route is stored as one path, and the collected signal data can be stored for each Access Point (AP) on each grid. Fig. 1 shows the procedures of the training phase. The signal strength information collected in the operation outlined above, needs to be processed for noise reduction as well as for the purposes of building a signal strength distribution model. The signal strength data collected from the APs is processed using an adaptive Kalman filtering algorithm to eliminate the noise and interference effects. In building the signal distribution model, interpolation is applied. The signal distribution at each point on the data gathering route is stored in the Radiomap database of the server computer.

\section{(2) Tracking phase}

In the tracking phase, signal data are processed in real-time by the filtering algorithm of the RSSI is compared with that stored in Radiomap. Based on this comparison, the optimal 
location is determined and marked on the map efficiently using color representative visualization approach.

\section{B. Grid Segment}

We present a Grid Segment Process to segmenting area in the building. The segment size is assumed in a tolerant distance where people can see other people clearly in that chosen distance. This assumption is used because we design our indoor location estimation system for using on disaster management for monitoring and finding people or user inside a hospital or group home in the normal situation or disaster situation. If we can determine the location of a user in the estimated grid position, this will enough to using such information by other people to finding or monitoring the user. Initial distance for grid size in this research is 5 meters. If the grid size with its error on an estimation is not accurate enough, we can extend the radio map into more detailed segment of the grid to achieve better accuracy. This can be done by using proposed color interpolation method explained in the next section. After creating grid segment layout for the building, then signal information is collected on each grid for each AP. We use median value from around 20 samples for each grid.

\section{Color Representation for Color Radiomap}

The received signal of each access point is converted into its color representative. This system uses 3 signals information from 3 different AP's based on triangulation approach. Each access point has its basic color that different each other. The three input $\mathrm{AP}\left(A P_{1}, A P_{2}, \& A P_{3}\right)$ using base color red, green and blue color respectively. The color map is based on signal strength information recorded from signal data collection process. The gradation of color is based on the HSL (Hue Saturation Luminance) value where Luminance is a function of the signal strength. Assuming $S S_{p x}$ is a variable for signal strength (in percentage) for the position of $x$ meter from the initial position. Then the color for the grid of $x$ meter distance is measured by these formulas:

$$
\begin{aligned}
& A P_{1} \rightarrow R G B(255,0,0) \rightarrow \operatorname{HSL}\left(0,240, f\left(L_{x}\right)\right) \\
& A P_{2} \rightarrow R G B(0,255,0) \rightarrow \operatorname{HSL}\left(80,240, f\left(L_{x}\right)\right) \\
& A P_{3} \rightarrow R G B(0,0,255) \rightarrow \operatorname{HSL}\left(160,240, f\left(L_{x}\right)\right) \\
& f\left(L_{x}\right)=240-\left(S S_{p x} * 120\right)
\end{aligned}
$$

where Equation 2 to 4 represent the color function for each AP's, the Equation 5 is the function to determine $L$ value on each grid $(x)$ position. Figure 2 show the illustration of the process on creating combination color radio map. First, the collected signal from each AP is filtered before converted into its gradation color map. Then create a new color radio map as a function of the sum from 3 different color radio maps. The sum of 3 color based on its RGB value that summary with OR operation.

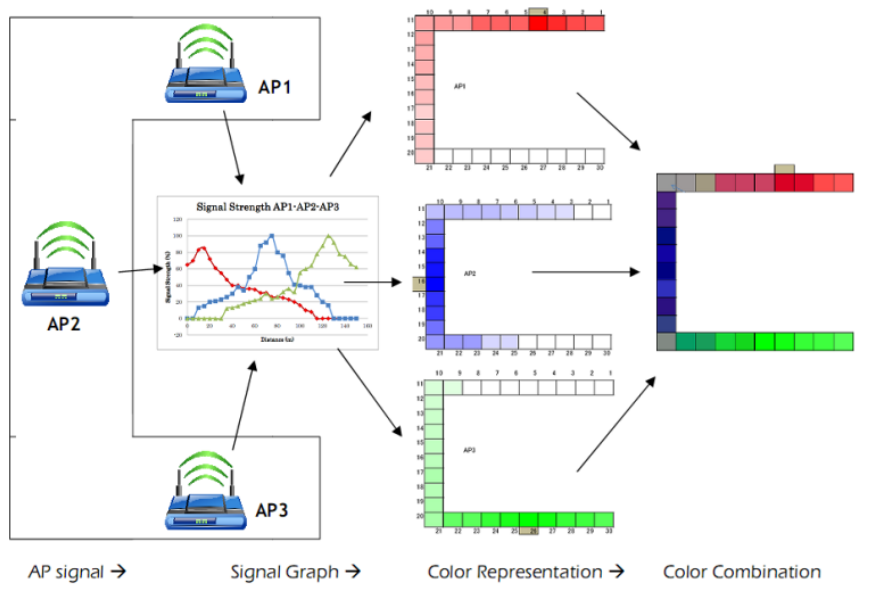

Fig. 2. Flow Diagram on creating Color Radio Map by color combination from 3 different AP signal strength.

\section{Interpolation with Color Grid Fusion}

Since the initial radio map with the initial grid size is not accurate enough, we improved the radiomap by interpolation. The effectiveness of this method is the interpolation using color information of each grid using grid fusion technique. We start with cell or grid size of 5 meters long with 2.5 widths (the corridor width). This initial grid size is determined into a number that low cost on offline training. The initial color radiomap will have the initial error also around 5 meters. Then, to improve the accuracy of the system; the initial map is improved to create a new map with detailed color. Initial grid size is fission into half size and the new value calculating by interpolation between two existing grid. Figure 3 show the illustration of grid fusion technique.
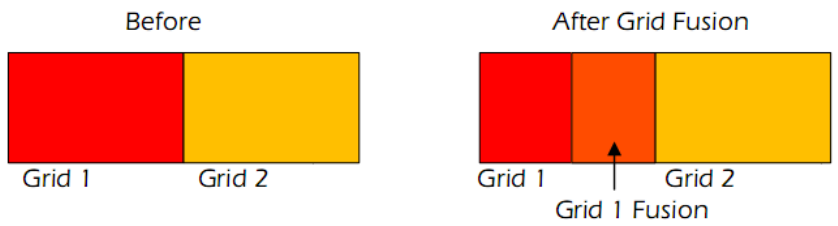

Fig. 3. Illustration of Grid Fusion for color interpolation

The fission of the grid is half size and the color is based on the RGB combination of two adjacent colors using the formula:

$$
\begin{aligned}
& \operatorname{Grid}_{1} \text { Fusion }(r, g, b)=\operatorname{Grid}_{1}\left(r_{1}, g_{1}, b_{1}\right)+\operatorname{Grid}_{2}\left(r_{2}, g_{2}, b_{2}\right) \\
& r=\left(r_{1}+r_{2}\right) / 2, g=\left(g_{1}+g_{2}\right) / 2 \text { and } b=\left(b_{1}+b_{2}\right) / 2,
\end{aligned}
$$

where $r$ is decimal value of Red, $g$ for Green and $b$ for Blue of the grid color. For example, if initial Grid 1 and Grid 2 has the RGB color is $\operatorname{Grid}_{1}(255,0,0)$, and $\operatorname{Grid}_{2}(255,102,0)$, then the Grid 1 Fusion become $(255,51,0)$. Interpolation for each adjacent grid generates a new grid with more detail color map as shown in Figure 7. The second stage of grid fusion is aim to create a more detail color radio map grid by using existing signal strength data from initial offline phase. The accuracy of detailed color radiomap then measured the investigate the accuracy. 


\section{E. User Location Estimation \& Visualization}

The user location determination process is based on the comparison between real-time online data and predefined offline color radiomap to find the minimum error between these two values on its color RGB value. Assume that online signal strength data from 3 AP's is converted into its HSL value then combined into one color using the same process like creating offline map, the RGB representative is $\left(r_{s}, g_{s}, b_{s}\right)$. Then the minimum error function is examined to all grid position $\operatorname{Grid}_{x}\left(r_{x}, g_{s}, b_{s}\right)$ to find the minimum error using minimum square error (MSE) formula:

$$
M S E=\left[\left(r_{x}-r_{s}\right)^{2}+\left(g_{x}-g_{s}\right)^{2}+\left(b_{x}-b_{s}\right)^{2}\right] / 3
$$

Indoor location estimation system is integrated with online real time monitoring systems. Using color combination technique for position estimation, the monitoring and visualizations of the estimate position becoming easier. Simple color masking technique using input color information as a representation of the combination of 3 signal strength of a user's device measurement at the moment, we can calculate and directly show the user position without using mean square error formula. The method for determine user position in a grid using color layering combination based on this algorithm:

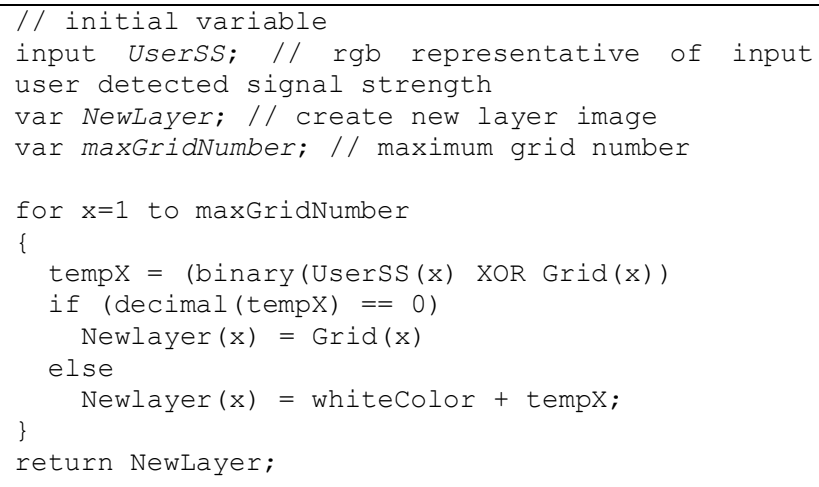

Fig. 4. Grid Masking Algorithm for efficient user tracking.

By using this masking algorithm, information about user estimation position automatically show on the new layer image only by masking user input data to color radiomap grid. This algorithm work for both online tracking user's position as well as on immediacy user position estimation. This approach is effective since no mathematical calculation for finding the MSE but only graphics processing that directly show the output of user position into the monitoring system.

\section{EXPERIMENTAL EVALUATION}

\section{A. System Testbed}

We performed the experiment in the third floor of the Engineering faculty Building 1, Saga University. This building has a layout like $\mathrm{C}$ alphabet as shown in Figure 2 with the total dimension in a rectangle is $150 \times 2.5$ meters. The building is equipped with $802.11 \mathrm{~b}$ wireless LAN environment. To form the radio map, the environment was modeled as a space of 30 cell locations in a grid of $5 \times 2.5$ meters each. This size is chosen as starting cell grid size, then interpolates it to make more detailed map with color radiomap interpolation.
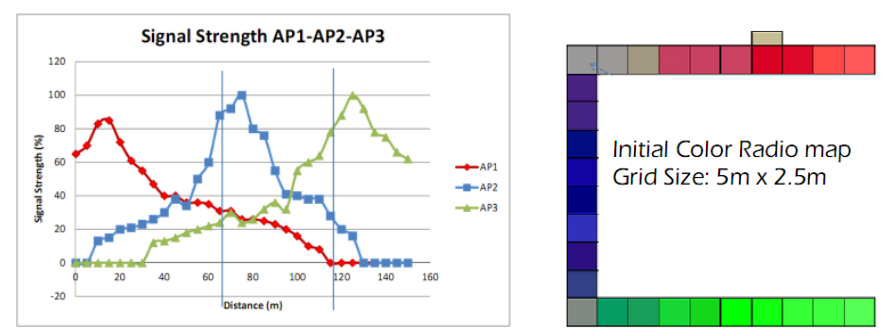

Fig. 5. Graph of Signal Strength Measured from 3 AP (left) and its combination color radiomap (right)

Since we deem that train data on too many locations on offline phase is impractical, we attempt to calibrate in few locations and interpolate all the other data on grid points by our model. In the experiment environment, we collected 1 point on the centre of each grid with sample number is 20 for each grid, and select the median value. The recorded signal strength information of each AP then translated to its color representation using Equation 1 to 4, to get the color radiomap. Finally, we combine the 3 color radiomap from different AP data into one map. The initial offline measurement results graph of training phase and the combination of color radio map is shown in Figure 5.

For evaluating the accuracy of proposed system, we conduct online tracking experiment and measure the results accuracy and errors. The evaluation phase use data from 25 random positions between 1 to 150 meters. To illustrate the experiment process we choose 6 position of user as initial online tracking position as shown in Figure 6; assume that measurement start from top right through the bottom right. The position of AP's is

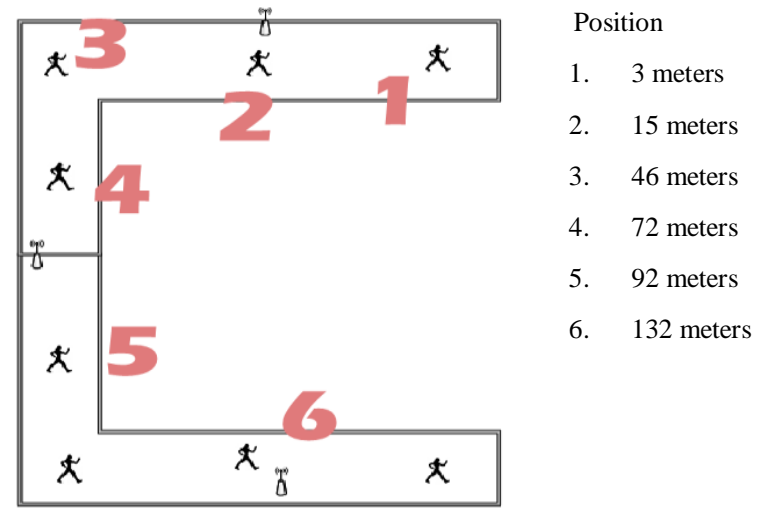

Fig. 6. Online tracking random sample position.

\section{B. Experiment Results}

The results of the experiments on the 6 random positions show that proposed system succeed to determine the user position by using information from measured signal strength from 3 AP's to the user device. Table 1 shows the results of estimated grid and the errors for the testing positions. Because the results are in the cell grid position which is a rectangle area, not a point position, the distance error is measured by taking the maximum of absolute number of the difference between the real positions to each grid border. Maximum difference between grid border size and real position is called as 
maximum error and the minimum difference between grid border point and the real position is called as minimum error. For example, first test position is 3 meter and the estimated grid is the grid number 1 which is located at 0 meters to 5 meters, so the maximum error is 3 meters and minimum error is 2 meters. The average error for 6 samples with initial color radio map is 3.5 meters. This error is quite enough for victim location estimator in disaster management system, but still not accurate enough as a precise indoor location estimation system. We can improve the accuracy by creating the new radiomap with smaller grid size using color radiomap interpolation method.

TABLE I. GRID ESTIMATION RESULTS FOR 6 SAMPLES WITH ERROR

\begin{tabular}{|c|c|c|c|c|}
\hline No & $\begin{array}{c}\text { Online Position } \\
\text { (meters) }\end{array}$ & Estimated Grid & $\begin{array}{c}\text { Min } \\
\text { Error } \\
\text { (meters) }\end{array}$ & $\begin{array}{c}\text { Max } \\
\text { Error } \\
\text { (meters) }\end{array}$ \\
\hline 1. & 3 & Grid $1[0 \mathrm{~m}-5 \mathrm{~m}]$ & 2 & 3 \\
\hline 2. & 15 & Grid 2 [15m-20m] & 0 & 5 \\
\hline 3. & 46 & Grid 10 [45m-50m] & 1 & 4 \\
\hline 4. & 72 & Grid 15 [70m-75m] & 2 & 3 \\
\hline 5. & 92 & Grid 19 [90m-95m] & 2 & 3 \\
\hline 6 & 132 & Grid 27 [130m-135m] & 3 & 3 \\
\hline
\end{tabular}

C. Efficiency and Accuracy of Grid Fusion

Initial color radiomap with 5 meter width of grid size, has minimum error 1.5 meters and maximum error 3.5 meters of the average error for 6 sample point. To improve the accuracy, we create a new map by reduce the grid size and interpolate the value from initial map. Two level grid fusions from 5 meters to 2.5 meters and from 2.5 meters to 1.25 meters size is created as shown in Figure 7 with the illustrate to show the color matching results. Figure 7 (bottom) also show the estimation position results for starting grid size and after interpolate into half size of the starting grid.

To evaluate the effectiveness and accuracy of proposed color grid interpolation method, we create another color radiomap start with grid size $8 \times 2.5$ meters then fission interpolate into 4 meters grid and 2 meters grid. Figure 8 show the graph of accuracy error from 2 color radiomap scheme for 25 samples from random position as online testing data. Radio Map with grid size $2.5 \times 2.5$ meters has average error 2.39 meters while radio map grid $C$ with grid size 1.25 x 2.5 meters has average error 1.59 meters. Improvement of color radio map with smaller grid size has decrease the accuracy error as shown in Figure 8. The experiments show that grid fission with color interpolation has significantly reduced the accuracy error. From the results, we found also that smaller initial cell grid size perform better than larger initial cell grid size.

The accuracy and effectiveness of our method is also compared with other previous method on indoor location estimation especially fingerprint and deterministic based method. Table 2 shows the comparison on the accuracy and the complexity for creating radiomap.
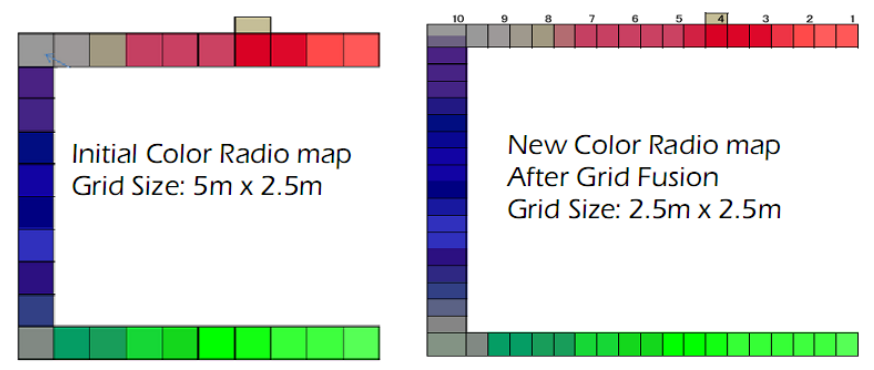

New Color Radio map

After Grid Fusion

Grid Size: $2.5 \mathrm{~m} \times 2.5 \mathrm{~m}$
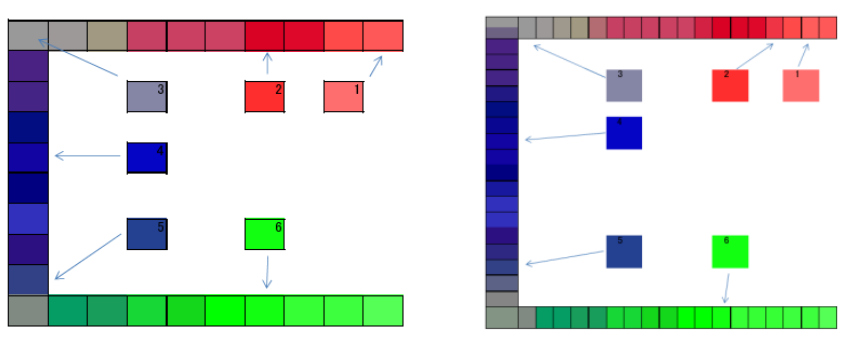

Fig. 7. Online tracking random sample position..
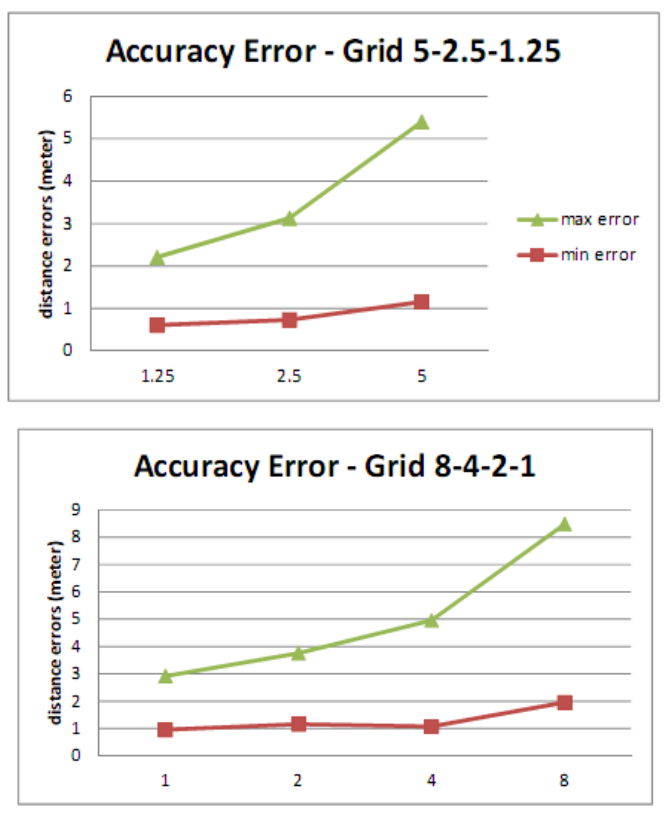

Fig. 8. Accuracy Error Graph of two map gradation. 5 meters scheme (top), and 8 meters scheme (bottom)

The accuracy means the distance error between real locations comparing to estimated results from the system. We use the mean error value (average of max error and min error) to compare the estimation error. Complexity means the effort on calibration process for creating radiomap or fingerprint database. The complexity is determined into 3 levels as follows: relatively high complexity, medium complexity, simple complexity. Comparing with other method, proposed color radiomap interpolation show high accuracy with simple complexity superior to RADAR one of the famed location estimation systems. Tzu Chieh [8] reported that they has 0.9076 meter on accuracy error but still in medium complexity on reducing calibration effort, while our method results can compete but with less complexity. Krumm [8] also show the 
simple complexity but the accuracy error still high above 3 meters error.

TABLE II. ACCURACY AND EFFICIENCY COMPARISON

\begin{tabular}{|c|l|c|c|}
\hline No & \multicolumn{1}{|c|}{ Method } & $\begin{array}{c}\text { Estimation Error } \\
\text { (meters) }\end{array}$ & Complexity \\
\hline 1. & RADAR [3] & 1.7588 & High \\
\hline 2. & Tzu Chieh [8] & 0.9076 & Medium \\
\hline 3. & J Krumm [5] & 3.75 & Simple \\
\hline 3. & Proposed Method & 1.108 & Simple \\
\hline
\end{tabular}

\section{CONCLUSION AND FUTURE WORK}

In this paper, we empirically study the effect of reducing the calibration effort on estimate the fingerprint radio map by interpolate new map with smaller grid size using color radiomap interpolation method. Both the offline and online phase is based on color combination and gradation interpolation method. Experiments show that creating new accurate map with interpolate unlabeled area using color information has reducing the calibration process while achieve high accuracy. Also, the determination of the user position can be done more effectively through the image processing integrated in monitoring and visualization systems.

We compare our system with some other famed location estimation system and demonstrate that our system perform simple complexity while gain high accuracy compare to others method.

In the future, we plan to improve this method to find the best grid size and implement it on user monitoring system for disaster management system. Moreover, we intend to test the validity of our proposed method in other complex building with many rooms and floor. The effect of using more or less than 3 $\mathrm{AP}$ is also considered for future investigation.

\section{REFERENCES}

[1] Seidel, S.Y. and T.S. Rapport, $914 \mathrm{MHz}$ Path Loss Prediction Model for Indoor Wireless Communications in Multifloored Buildings. IEEE Transactions on Antennas and Propagation, 1992. 40(2): p. 207-217.

[2] Wolfle, G. and F.M. Landstorfer. Dominant Paths for the Field Strength Prediction. in 48th IEEE Vehicular Technology Conference (VTC). 1998. Ottawa, Ontario, Canada.

[3] P. Bahl, V.N. Padmanabhan, RADAR: an in-building RF-based user location and tracking system, INFOCOM 2000. 19th Annual Joint Conference of the IEEE Computer and Communications Societies, Proceedings IEEE 2 (2000) 775-784 vol.772.

[4] Xiaoyong Chai, Qiang Yang, Reducing the Calibration Effort for Probabilistic Indoor Location Estimation, IEEE Transactions on Mobile Computing, v.6 n.6, p.649-662, June 2007

[5] J. Krumm and J.C. Platt, "Minimizing Calibration Effort for an Indoor 802. 11 Device Location Measurement System," technical report, Microsoft Research, 2003.
[6] A. Bose, F. Chuan Heng, A Practical Path Loss Model for Indoor WiFi Positioning Enhancement, Information, Communications \& Signal Processing, $20076^{\text {th }}$ International Conference, 2007, pp. 1-5.

[7] Y. Chen, X. He, Contribution of pseudolite observations to GPS precise surveys, KSCE Journal of Civil Engineering 12 (1) (2008) 31-36.

[8] Tzu-Chieh Tsai; Cheng-Lin Li, "Reducing Calibration Effort for WLAN Location System using Segment Technique with Autocorrelation," Communications and Networking in China, 2006. ChinaCom '06. First International Conference on, vol., no., pp.1-5, 2527 Oct. 2006

[9] Y.K. Cho, J.-H. Youn, N. Pham, Performance tests for wireless real-time localization systems to improve mobile robot navigation in various indoor environments, in: C. Balaguer, M. Abderrahim (Eds.), Robotics and Automation in Construction, InTech Education and Publishing, 2008, pp. 355-372.

[10] Y. Fukuju,M. Minami, H.Morikawa, T. Aoyama, DOLPHIN: An Autonomous Indoor Positioning System in Ubiquitous Computing Environment, Proceedings of the IEEE Workshop on Software Technologies for Future Embedded Systems, IEEE Computer Society, 2003.

[11] C. Hu, W. Chen, Y. Chen, D. Liu, Adaptive Kalman filtering for vehicle navigation, Journal of Global Positioning Systems 2 (1) (2003) 6.

[12] J. Yim, C. Park, J. Joo, S. Jeong, Extended Kalman filter for wireless LAN based indoor positioning, Decision Support System 45 (4) (2008) 960-971.

[13] W.-S. Jang, M.J. Skibniewski, A wireless network system for automated tracking of construction materials on project sites, Journal of Civil Engineering and Management 14 (1) (2008) 9.

[14] H.M. Khoury, V.R. Kamat, Evaluation of position tracking technologies for user localization in indoor construction environments, Automation in Construction 18 (4) (2009) 444-457.

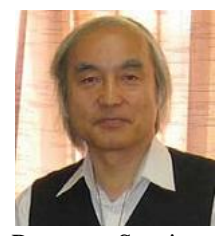

AUTHORS PROFILE

Kohei Arai, He received BS, MS and PhD degrees in 1972, 1974 and 1982, respectively. He was with The Institute for Industrial Science and Technology of the University of Tokyo from April 1974 to December 1978 and also was with National Space Development Agency of Japan from January, 1979 to March, 1990. During from 1985 to 1987, he was with Canada Centre for Remote Sensing as a Post Doctoral Fellow of National Science and Engineering Research Council of Canada. He moved to Saga University as a Professor in Department of Information Science on April 1990. He was a councilor for the Aeronautics and Space related to the Technology Committee of the Ministry of Science and Technology during from 1998 to 2000. He was a councilor of Saga University for 2002 and 2003. He also was an executive councilor for the Remote Sensing Society of Japan for 2003 to 2005. He is an Adjunct Professor of University of Arizona, USA since 1998. He also is Vice Chairman of the Commission A of ICSU/COSPAR since 2008. He wrote 26 books and published 327 journal papers.

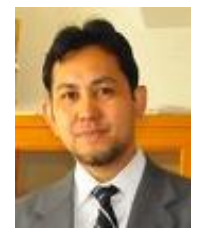

Herman Tolle, He graduated Bachelor degree in Electrical Engineering from Brawijaya University, Malang in 1998, and also graduated Master degree in Telecommunication Information System from Bandung Institute of Technology (ITB), Bandung in 2002. He received his $\mathrm{PhD}$ degrees in 2011 from Saga University. He is with Software Engineering Department of Brawijaya University from 2002 to present. He is now a post Doctoral fellow in Department of Information Science, Saga University Japan. He has major concern of research in image analysis, mobile computing, content adaptation and web engineering. 